\title{
Correlation between serum YKL-40 levels and albuminuria in type 2 diabetes
}

\author{
J.Y. Han ${ }^{1}$, X.Y. Ma', L.J. Yu' ${ }^{1}$, Y. Shao ${ }^{2}$ and Q.Y. Wang ${ }^{2}$ \\ ${ }^{1}$ The Geriatrics Department, \\ The First Affiliated Hospital of China Medical University, Shenyang, \\ Liaoning Province, China \\ ${ }^{2}$ The Endocrine Department, \\ The First Affiliated Hospital of China Medical University, Shenyang, \\ Liaoning Province, China \\ Corresponding author: Q.Y. Wang \\ E-mail: wqycmu@163.com
}

Genet. Mol. Res. 14 (4): 18596-18603 (2015)

Received April 25, 2015

Accepted July 26, 2015

Published December 28, 2015

DOI http://dx.doi.org/10.4238/2015.December.28.7

ABSTRACT. We explored the correlation between serum YKL-40 levels and albuminuria in type 2 diabetes mellitus (T2DM) and its clinical significance. This study used a cross-sectional survey method. According to the American Diabetes Association 2007 Clinical Practice Recommendations, 738 patients with T2DM were divided into three groups: a normoalbuminuria group [albumin-to-creatinine ratio (ACR) $<30$ $\mu \mathrm{g} / \mathrm{mg}, \mathrm{N}=360$ ], a microalbuminuria group (ACR 30-300 $\mu \mathrm{g} / \mathrm{mg}, \mathrm{N}=246$ ), and a macroalbuminuria group ( $A C R \geq 300 \mu \mathrm{g} / \mathrm{mg}, \mathrm{N}=332$ ). The serum YKL-40 levels were determined by a quantitative sandwich enzyme-linked immunosorbent assay in all the cases and in 210 control subjects. Serum YKL-40 levels were significantly higher in the T2DM group vs the control group $(P<0.05)$, the macroalbuminuria group vs the microalbuminuria group $(P<0.05)$, and the microalbuminuria group $v s$ the normoalbuminuria group $(P<0.05)$. Serum $Y K L-40$ levels correlated with $A C R$ in all 
participants. Significant correlation of YKL-40 was found with ACR, 2-h plasma glucose, glycated hemoglobin, fasting blood glucose, homeostatic model assessment of insulin resistance index, systolic blood pressure, duration, diastolic blood pressure, age, triglycerides, and high-density lipoprotein cholesterol (r-values: $0.713,0.524,0.515,0.467,0.438,0.409$, $0.407,0.374,0.112,0.097$, and -0.123 , respectively). ACR correlated with serum YKL-40 levels (Beta $=0.555, P<0.001)$. YKL-40 may be involved in the occurrence and development of diabetic nephropathy and would be useful as a new marker for the disease.

Key words: Diabetic nephropathy; Type 2 diabetes mellitus

\section{INTRODUCTION}

Diabetic nephropathy (DN) is one of the microvascular complications of diabetes mellitus and is an important cause of end-stage renal disease, with high morbidity and mortality. $\mathrm{YKL}-40$ is an inflammatory glycoprotein. It is a $40-\mathrm{kDa}$ heparin- and chitin-binding lectin and a member of the mammalian chitinase-like protein family, known as cartilage glycoprotein-39 or chitinase-3-like protein 1. YKL-40 is secreted by a variety of human cells. Several studies have demonstrated that the plasma levels of $\mathrm{YKL}-40$ increase significantly with increasing severity of albuminuria in type 1 diabetes mellitus (T1DM) (Rathcke et al., 2009). Moreover, YKL-40 has also been found to increase more in T2DM patients than in a control group, and it is correlated with mortality (Thomsen et al., 2010; Persson et al., 2012). Here, we attempted to evaluate the association between plasma YKL-40 levels and different levels of albuminuria in type 2 DN.

\section{MATERIAL AND METHODS}

\section{Subjects}

In total, 738 T2DM patients were enrolled at the First Affiliated Hospital of China Medical University between June 2012 and July 2014 (375 males and 363 females). We excluded patients based on the following criteria: those with heart, liver, or other kidney diseases, or any kind of infectious disease; those with abnormal renal function status, those that had experienced diabetic ketoacidosis or recent hyperosmolar coma; those with a history of renin-angiotensin system inhibitor usage over the previous three months; and those receiving insulin treatment. The 738 diabetic patients were divided into three groups according to albuminuria levels, based on albumin-tocreatinine ratios (ACR): a normoalbuminuria group ( $A C R<30 \mu \mathrm{g} / \mathrm{mg}, \mathrm{N}=260$ ); a microalbuminuria group (ACR 30-300 $\mu \mathrm{g} / \mathrm{mg}, \mathrm{N}=246$ ); and a macroalbuminuria group (ACR $\geq 300 \mu \mathrm{g} / \mathrm{mg}, \mathrm{N}=$ 232). All control subjects $(N=210)$ were recruited from the medical center of the First Affiliated Hospital of China Medical University, and exclusions were based on the following criteria: diabetes, hypertension, heart disease, lipid metabolism disorders, kidney disease, liver disease, and other endocrine and metabolic diseases. 
Informed consent was obtained from each patient before the study began. Diabetes was diagnosed according to the American Diabetes Association 2007 Clinical Practice Recommendations.

\section{Measurements}

Venous blood ( $5 \mathrm{~mL}$ ) was taken from the participants following 8-10 h overnight fasting, using pyrogen- and endotoxin-free disposable BD Biosciences Vacutainers (UK). The blood samples were then centrifuged at $2000 \mathrm{~g}$ for $10 \mathrm{~min}$ and the serum was collected. We kept the serum at $-70^{\circ} \mathrm{C}$ until required.

We recorded the following clinical parameters of all the subjects: gender, age, disease duration, systolic blood pressure (SBP), diastolic blood pressure (DBP), weight, height, waist circumference (WC), hip circumference, smoking history, alcohol consumption, renal function (serum creatinine and blood urea nitrogen), liver function, blood uric acid (BUA), lipids (low-density lipoprotein cholesterol (LDL-C), high-density lipoprotein cholesterol (HDL-C), total cholesterol (TC), triglycerides (TG), glycated hemoglobin $(\mathrm{HbA} 1 \mathrm{c})$, fasting blood glucose and 2-h plasma glucose ( $2 \mathrm{hPG}$ ) oral glucose tolerance test, and islet function results and history of related complications. (We considered macrovascular complications according to a lower extremity artery ultrasound examination, and atherosclerotic stenosis was considered to have major vascular complications; peripheral neuropathy was assessed based on electromyography results; diabetic retinopathy was assessed based on fundus examination and diagnosis by ophthalmic doctors.) Creatinine clearance $(\mathrm{CCr})$ was calculated as follows: $[\mathrm{CCr}=(140-$ age $) \times$ weight $(\mathrm{kg}) / 72 \times$ serum creatinine (Scr) (mg/dL) (x 0.85 for females)]. The homeostasis model assessment evaluation of insulin resistance (HOMA-IR) index was calculated as follows: (HOMA-IR = FPG x FINS/22.5) (FPG is fasting plasma glucose and FINS is fasting insulin).

We tested the level of serum YKL-40 by enzyme-linked immunosorbent assay (ELISA) (purchased from the Bio-Technology Co. Ltd., USA) (Table 1).

\section{Statistical analyses}

Statistical analyses were performed using SPSS version 17.0. Data are reported as means \pm SD for normally distributed values, median (interquartile range) for nonparametric values, and proportion for categorical variables. Distribution of continuous variables was examined, and logarithm-transformed values of non-normal distribution variables were used for conducting analyses. Differences between the groups were analyzed by analysis of variance for normal distribution and by non-parametric rank-test for non-normal distribution, followed by the Kruskal-Wallis test for nonparametric values. The $X^{2}$ test was used to analyze categorical data as appropriate. To determine the clinical parameters correlated with serum YKL-40, multiple linear regression analysis was examined. Finally, we conducted multivariate regression analyses with urinary albuminuria and serum YKL-40 as dependent variables. Several models were gradually built to adjust for several confounding factors; we ultimately chose model 11. P-values of less than 0.05 were considered statistically significant. 
Table 1. Comparison between groups: general information and biochemical data.

\begin{tabular}{|c|c|c|c|c|c|}
\hline & \multirow[t]{2}{*}{ Control group } & \multicolumn{3}{|c|}{ T2DM group } & \multirow[t]{2}{*}{$P$ value } \\
\hline & & Normoalbuminuria & Microalbuminuria & Macroalbuminuria & \\
\hline $\mathrm{N}$, total & 210 & 260 & 246 & 232 & \\
\hline Male (\%) & 48.4 & 50.7 & 51.7 & 47.8 & 0.854 \\
\hline Age (years) & $53.40 \pm 4.28$ & $52.83 \pm 4.30$ & $53.93 \pm 4.56$ & $53.93 \pm 4.22^{\mathrm{a}}$ & 0.086 \\
\hline DM duration (years) & - & $4.70 \pm 3.05$ & $4.14 \pm 2.83$ & $4.81 \pm 3.07^{e}$ & $<0.001$ \\
\hline $\mathrm{HbA} 1 \mathrm{c}(\%)$ & $5.40 \pm 0.42$ & $8.25 \pm 1.16^{\mathrm{b}}$ & $8.52 \pm 1.06^{b}$ & $8.30 \pm 1.07^{b}$ & $<0.001$ \\
\hline BMI $\left(\mathrm{kg} / \mathrm{m}^{2}\right)$ & $23.45 \pm 2.37$ & $23.99 \pm 2.99$ & $24.29 \pm 2.73$ & $24.09 \pm 2.58$ & 0.100 \\
\hline$W C(\mathrm{~cm})$ & $80.44 \pm 9.90$ & $87.86 \pm 11.33^{b}$ & $89.05 \pm 10.07^{b}$ & $95.58 \pm 6.81^{\text {bdf }}$ & $<0.001$ \\
\hline WHR & $0.8 \pm 0.6$ & $0.9 \pm 0.1^{\mathrm{b}}$ & $0.9 \pm 0.5^{\mathrm{b}}$ & $0.9 \pm 0.7^{\text {bdf }}$ & $<0.001$ \\
\hline SBP (mmHg) & $127.00 \pm 6.32$ & $121.96 \pm 13.20$ & $130.93 \pm 13.76^{d}$ & $145.58 \pm 20.73^{\text {bdf }}$ & $<0.001$ \\
\hline $\mathrm{DBP}(\mathrm{mmHg})$ & $75.64 \pm 7.17$ & $79.46 \pm 9.52$ & $82.74 \pm 9.58$ & $86.29 \pm 9.74$ & $<0.001$ \\
\hline $\mathrm{FBG}(\mathrm{mM})$ & $5.11 \pm 0.47$ & $9.57 \pm 2.88^{\mathrm{b}}$ & $9.74 \pm 2.75^{\mathrm{b}}$ & $9.94 \pm 1.87^{\mathrm{b}}$ & $<0.001$ \\
\hline $2 \mathrm{hPG}(\mathrm{mM})$ & $6.74 \pm 0.82$ & $18.54 \pm 4.49^{b}$ & $20.61 \pm 5.22^{\mathrm{b}}$ & $17.27 \pm 5.79^{\text {bf }}$ & $<0.001$ \\
\hline LDL-C (mM) & $2.95 \pm 0.90$ & $2.85 \pm 0.92$ & $2.88 \pm 0.81$ & $2.97 \pm 0.93$ & 0.690 \\
\hline HDL-C (mM) & $1.26 \pm 0.30$ & $1.20 \pm 0.29$ & $1.18 \pm 0.32$ & $1.22 \pm 0.32$ & 0.171 \\
\hline $\mathrm{TG}(\mathrm{mM})$ & $1.37 \pm 0.60$ & $1.48 \pm 0.99$ & $1.52 \pm 0.98$ & $1.45 \pm 0.91$ & 0.603 \\
\hline $\mathrm{TC}(\mathrm{mM})$ & $4.74 \pm 1.12$ & $4.87 \pm 1.04$ & $4.92 \pm 1.43$ & $5.00 \pm 1.25$ & 0.412 \\
\hline $\mathrm{BUA}(\mathrm{mM})$ & $340.78 .6 \pm 55.2$ & $350.31 \pm 65.55$ & $348.55 \pm 65.74$ & $351.61 \pm 68.39^{\text {df }}$ & 0.572 \\
\hline $\operatorname{Scr}(\mu \mathrm{M})$ & $74.25 \pm 11.98$ & $74.06 \pm 13.93$ & $74.44 \pm 12.58$ & $76.7 \pm 11.8$ & 0.291 \\
\hline $\mathrm{CCr}(\mathrm{mL} / \mathrm{min})$ & $89.30 \pm 18.69$ & $89.91 \pm 19.72$ & $92.19 \pm 21.7$ & $86.50 \pm 17.03$ & 0.113 \\
\hline $\operatorname{ACR}(\mu \mathrm{g} / \mathrm{mg})$ & $10.4(7.5-15)$. & $13.5(7.34-17.58)$ & $74.28(45.54-110.12)$ & $782.52(409.51-2130.17)$ & $<0.001$ \\
\hline YKL-40 (ng/mL) & $27.6(24.5-29.3)$ & $44.6(41.1-48.8)^{\mathrm{b}}$ & $67.5(61.9-70.7)^{\text {bd }}$ & $72.5(60.5-76.3)^{\mathrm{bdf}}$ & $<0.001$ \\
\hline FINS (mIU/L) & - & $6.93(5.26-10.82)$ & $9.94(8.12-16.58)^{d}$ & $17.59(9.11-29.17)^{d f}$ & $<0.001$ \\
\hline 2hPINS (mIU/L) & - & $22.71(15.37-28.9)$ & $22.94(16.1-38.07)$ & $39.89(19.85-86.3)^{\mathrm{df}}$ & $<0.001$ \\
\hline HOMA-IR & - & $3.40(2.09-3.86)$ & $4.53(3.03-6.49)^{d}$ & $7.42(4.77-10.81)^{\mathrm{df}}$ & $<0.001$ \\
\hline \multicolumn{6}{|l|}{ Life history } \\
\hline Smoking (\%) & 10.9 & 32.5 & 36.3 & 29.6 & 0.399 \\
\hline Drinking (\%) & 9.1 & 21.2 & 27.4 & 18.2 & 0.296 \\
\hline \multicolumn{6}{|l|}{ History of complications } \\
\hline Hypertension (\%) & 0 & 12.5 & 28.1 & 40.9 & $<0.001$ \\
\hline Macrovascular (\%) & 0 & 3.75 & 8.9 & 24.2 & $<0.001$ \\
\hline Peripheral neuropathy (\%) & 0 & 6.5 & 13.7 & 33.3 & $<0.001$ \\
\hline Diabetic retinopathy (\%) & 0 & 13.75 & 20.55 & 30.3 & 0.001 \\
\hline
\end{tabular}

$\mathrm{DM}=$ diabetes mellitus; $\mathrm{HbA} 1 \mathrm{c}=$ glycated hemoglobin; $\mathrm{BMI}=$ body mass index; $\mathrm{WC}=$ waist circumference; $\mathrm{WHR}=$ waist-to-hip ratio; $\mathrm{SBP}=$ systolic blood pressure; $\mathrm{DBP}=$ diastolic blood pressure; $\mathrm{FBG}=$ fasting blood glucose; $2 \mathrm{hPG}$ = 2-h plasma glucose; LDL-C = low-density lipoprotein cholesterol; $\mathrm{HDL}-\mathrm{C}=$ high-density lipoprotein cholesterol; $\mathrm{TG}=$ triglycerides; $\mathrm{TC}=$ total cholesterol; $\mathrm{BUA}=$ blood uric acid; $\mathrm{Scr}=$ serum creatinine; $\mathrm{CCr}=$ creatinine clearance; $\mathrm{ACR}=$ albumin-to-creatinine ratio; FINS = fasting insulin; $2 \mathrm{hPINS}=2$-h postprandial insulin; HOMA-IR = homeostasis model assessment evaluation of insulin resistance. Compared with the control group ${ }^{\mathrm{a}} \mathrm{P}<0.05$, ${ }^{\mathrm{b}} \mathrm{P}<0.01$. Compared with normoalbuminuria group ${ }^{\mathrm{C}} \mathrm{P}<0.05,{ }^{\mathrm{d}} \mathrm{P}<0.01$. Compared with microalbuminuria group, ${ }^{\mathrm{e}} \mathrm{P}<0.05,{ }^{\mathrm{f}} \mathrm{P}<0.01$.

\section{RESULTS}

The groups of T2DM were closely matched in terms of age, gender, and duration of diabetes. We found statistically significant differences in median serum YKL-40 levels between the different groups $(P<0.05)$. Moreover, serum YKL-40 levels were significantly higher in the T2DM group vs the control group $(P<0.05)$, the macroalbuminuria group vs the microalbuminuria group $(P<0.05)$, and the microalbuminuria group vs the normoalbuminuria group $(P<0.05)$. Statistical differences were found in SBP, DBP, waist-to-hip ratio (WHR), 2hPG, HOMA-IR, and FINS among the T2DM groups with different rates of urinary albumin excretion $(P<0.05)$. We found significant differences in WC, WHR, FBG, and 2hPG between the control group and the T2DM groups $(P<0.05)$. However, there was no significant difference among the different T2DM groups. We then used the $X^{2}$ test to analyze differences among T2DM complications and associated complications. The results were as follows: hypertension $\left(X^{2}=72.197, P<0.001\right)$, macrovascular complications $\left(X^{2}=52.056, P<0.001\right)$, 
peripheral neuropathy $\left(X^{2}=68.823, P<0.001\right)$, and diabetic retinopathy $\left(X^{2}=41.986, P<0.001\right)$.

Table 2 reveals that the median and quartiles serum YKL-40 levels gradually increased. We conducted multivariate regression analyses and the results showed that ACR $(r=0.713, P<$ $0.05), 2 \mathrm{hPG}(r=0.524, \mathrm{P}=0.015)$, HbA1c $(r=0.515, \mathrm{P}<0.05)$, FBG $(r=0.467, \mathrm{P}<0.05)$, HOMAIR $(r=0.438, P=0.001)$, SBP $(r=0.409, P<0.05)$, duration $(r=0.407, P<0.05)$, DBP $(r=0.374$, $P<0.05)$, age $(r=0.112, P=0.009)$, TG $(r=0.097, P=0.024)$, and HDL-C $(r=-0.123, P=0.004)$ correlated with $\mathrm{YKL}-40$, and the correlation still existed even after excluding each complication. By multivariate regression analyses with $\mathrm{YKL}-40$ as a dependent variable and other relevant clinical data as covariates, the correlation was confirmed again among the various clinical data. Moreover, ACR influenced the serum level of YKL-40 at the maximum value (Beta $=0.555, P<0.001)$.

Table 2. Correlation between YKL-40 and clinical data.

\begin{tabular}{lcr}
\hline & Correlation coefficient & P value \\
\hline ACR & 0.713 & $<0.001$ \\
2hPG & 0.524 & 0.015 \\
HbA1c & 0.515 & $<0.001$ \\
FBG & 0.467 & $<0.001$ \\
HOMA-IR & 0.438 & 0.001 \\
SBP & 0.409 & $<0.001$ \\
Duration & 0.407 & $<0.001$ \\
DBP & 0.374 & $<0.001$ \\
Age & 0.112 & 0.009 \\
TG & 0.097 & 0.024 \\
HDL-C & -0.123 & 0.004 \\
\hline
\end{tabular}

ACR = albumin-to-creatinine ratio; $2 \mathrm{hPG}=$ 2-h plasma glucose; $\mathrm{HbA} 1 \mathrm{c}=$ glycated hemoglobin; $\mathrm{FBG}=$ fasting blood glucose; HOMA-IR = homeostasis model assessment evaluation of insulin resistance; SBP = systolic blood pressure; $\mathrm{DBP}=$ diastolic blood pressure; TG = triglycerides; HDL-C = high-density lipoprotein cholesterol.

From the final model, we produced the regression equation:

$\wedge y=-26.136+0.009 X_{1}+0.77 X_{2}+1.433 X_{3}+0.035 X_{4}+2.727 X_{5}+0.158 X_{6}+0.4 X_{7}-$
$0.095 X_{8}+0.444 X_{9}-0.81 X_{10}-0.129 X_{11}($ see Table 3).

Table 3. Multivariate regression of YKL-40 and clinical data.

\begin{tabular}{|c|c|c|c|c|c|}
\hline & \multicolumn{2}{|c|}{ Unstandardized coefficients } & \multirow{2}{*}{$\begin{array}{c}\text { Standardized coefficients } \\
\text { Beta }\end{array}$} & \multirow[t]{2}{*}{$t$-value } & \multirow[t]{2}{*}{$P$ value } \\
\hline & $B$ & Standard error & & & \\
\hline Constant & -26.136 & 7.730 & & -3.381 & 0.001 \\
\hline $\operatorname{ACR}\left(X_{1}\right)$ & 0.009 & 0.000 & 0.555 & 24.476 & $<0.001$ \\
\hline $2 \mathrm{hPG}\left(\mathrm{X}_{2}\right)$ & 0.770 & 0.116 & 0.245 & 6.617 & $<0.001$ \\
\hline HOMA-IR $\left(\mathrm{X}_{3}\right)$ & 1.433 & 0.163 & 0.276 & 8.797 & $<0.001$ \\
\hline $\operatorname{BUA}\left(X_{4}\right)$ & 0.035 & 0.007 & 0.104 & 5.100 & $<0.001$ \\
\hline $\mathrm{HbA1c}\left(\mathrm{X}_{5}\right)$ & 2.727 & 0.493 & 0.199 & 5.533 & $<0.001$ \\
\hline $\operatorname{SBP}\left(X_{6}\right)$ & 0.158 & 0.033 & 0.126 & 4.856 & $<0.001$ \\
\hline Age $\left(X_{7}\right)$ & 0.400 & 0.098 & 0.082 & 4.068 & $<0.001$ \\
\hline 2hPINS $\left(X_{8}\right)$ & -0.095 & 0.025 & -0.120 & -3.777 & $<0.001$ \\
\hline Duration $\left(\mathrm{X}_{9}\right)$ & 0.444 & 0.150 & 0.067 & 2.951 & 0.003 \\
\hline FPG $\left(X_{10}\right)$ & -0.810 & 0.285 & -0.112 & -2.841 & 0.005 \\
\hline $\operatorname{DBP}\left(X_{11}\right)$ & -0.129 & 0.054 & -0.059 & -2.364 & 0.018 \\
\hline
\end{tabular}

$\mathrm{ACR}=$ albumin-to-creatinine ratio; $2 \mathrm{hPG}=2$-h plasma glucose; $\mathrm{HOMA}-\mathrm{IR}=$ homeostasis model assessment evaluation of insulin resistance; $\mathrm{BUA}=$ blood uric acid; $\mathrm{HbA} 1 \mathrm{c}=$ glycated hemoglobin; $\mathrm{SBP}=$ systolic blood pressure; $2 \mathrm{hPINS}=$ 2-h postprandial insulin; FBG = fasting blood glucose; DBP = diastolic blood pressure. 


\section{DISCUSSION}

Microalbuminuria is an important indicator of the occurrence and progression of DN. It has now been confirmed that many inflammatory cytokines and cell signaling pathways have a relationship with DN (Horbinski et al., 2010; Cohen-Bucay and Viswanathan, 2012; Ogawa and Makino, 2012; Kanasaki et al., 2013; Moresco et al., 2013). Microalbuminuria is not, however, an ideal indicator of DN because there are renal pathological changes and progressive renal functions present during impaired glucose tolerance that are not reflected in urinary albumin levels. Therefore, there is a need for a convenient indicator that can detect DN at an early stage.

Human cartilage glycoprotein-39 (HCgp39), also known as chitinase-3-like protein 1, is a mammalian enzyme-like protein that is similar to a family of proteins found in crustaceans. The protein is also called YKL-40 because its amino terminus comprises a peptide chain starting with the three amino acids tyrosine $(\mathrm{Y})$, lysine $(\mathrm{K})$, and leucine $(\mathrm{L})$, and it has a relative molecular mass of approximately $40 \mathrm{kDa}$ (Rathcke et al., 2009). YKL-40 is an inflammatory glycoprotein that can promote cell chemotaxis, adhesion, and migration, and it has a role in endothelial dysfunction. Studies have shown that YKL-40 mRNA expression is highly regulated in a model of atherosclerosis, especially macrophage infiltration. Macrophage infiltration could promote the expression of YKL-40 mRNA, which is considered atherosclerosis in early lesions. (Batinic et al., 2012; Ma et al., 2012). In the past few years, it has been demonstrated that elevated levels of YKL-40 are correlated with the incidence and mortality of cardiovascular disease. Kastrup et al. (2009) have also proved that the level of YKL-40 in coronary atherosclerotic heart disease is an independent risk factor. CD68 mRNA in obese patients is higher than in normal people, and it has been proven that there is a correlation between YKL-40 and CR68 mRNA levels. Therefore, we can speculate that YKL40 is involved in immune and inflammatory responses in adipocytes (El-Mesallamy et al., 2011; Chakaroun et al., 2012).

Microalbuminuria is considered a risk factor for cardiovascular disease in diabetic patients. Many studies have shown that serum levels of $Y K L-40$ in diabetic patients are higher than in a control group. Rathcke et al. (2009) proved that YKL-40 had a correlation with type 1 diabetic nephropathy, and suggested a role of YKL-40 in the occurrence of microvascular complications during renal vascular damage. Thus, they considered that YKL-40 might be useful as an independent marker (Nielsen et al., 2008; Rathcke et al., 2009).

In this study, there were statistically significant differences between WC, WHR, FBG, and $2 \mathrm{hPG}$ in the control group and the T2DM patients $(\mathrm{P}<0.001)$, while there were no statistically significant differences among the T2DM groups. Gender, age, BMI, LDL-C, HDL-C, TG, TC, BUA, $\mathrm{Scr}$, and $\mathrm{CCr}$ were not significantly different in T2DM patients with different urinary albumin excretion rates in each group. Therefore, we ruled out the differences among these indicators in the T2DM groups, and proved the correlation between the level of YKL-40 and urinary albumin. Using linear correlation analysis, we found there was significant intercorrelation between $Y K L-40$ and $A C R$, 2hPG, HbA1c, FBG, HOMA-IR, SBP, duration, DBP, age, TG, and HDL-C. Regression coefficients showed that YKL-40 positively correlated with ACR and HOMA-IR, which were independent factors leading to elevated YKL-40 (correlation coefficients were $r=0.713, P<0.001$, and $r=0.438, P<$ 0.001 , respectively).

Our conclusion is consistent with that of a previous study (Lee et al., 2012). However, our study differed from that of Røndbjerg et al. (2011) in that we also investigated HOMA-IR, which is an indicator of insulin resistance and found that it was positively correlated with YKL-40 levels 
$(r=0.438, P<0.001)$. The pathophysiology characteristics of T2DM are mainly insulin resistance and lack of insulin secretion. It has been confirmed that obesity in T2DM patients is associated with serum levels of YKL-40. Insulin resistance usually exists in obese patients; hence, we speculated that YKL-40 might be correlated with it. It has been confirmed that insulin resistance plays a significant role in numerous conditions including pathological entities such as T2DM and obesity, and physiological states like high intake of saturated fat and physical inactivity. Under the condition of insulin resistance, glomerular efferent arteries contract excessively because of weakened vasodilator effect, which leads to increased blood volume, glomerular capillary hydrostatic pressure, and urinary albumin, thereby aggravating diabetic nephropathy damage. As with previous studies, we excluded hypertensive patients who had a history of treatment with angiotensin-converting enzyme inhibitors or angiotensin receptor blockers in all T2DM groups. These two classes of antihypertensive drugs can change the ACR by affecting the renin-changing renal aldosterone system, and reducing macroporous and non-selective protein leakage, thereby reducing urinary protein. Because these drugs affect the grouping criteria, we eliminated their influence in our study because it has been proved that the level of $\mathrm{YKL}-40$ is associated with the ACR, and we estimated that they might also affect the level of YKL-40. In contrast to the study by Brix et al. (2011), we included a control group to prove that serum YKL-40 levels were significantly different between T2DM patients and the control group $(P<$ 0.001). Correlation analysis and stepwise multiple regression revealed a positive correlation between YKL-40 and ACR, and ACR is an independent factor that leads to elevated YKL-40. YKL-40 is an inflammation and endothelial injury marker, and therefore it further confirms the pathogenesis of the DN inflammation hypothesis. Our study also found that other groups of diabetic complications, such as macrovascular complications $(P<0.001)$, diabetic neuropathy $(P$ $<0.001)$, and diabetic retinopathy $(P<0.001)$, were significantly correlated with an increase in $A C R$, indicating that some diabetic complications occur in parallel, but the relationship between other complications and YKL-40 requires further study.

We conclude from our data that the serum levels of YKL-40 in T2DM patients were significantly higher than in normal patients, and the levels of YKL-40 gradually increased with increasing urinary protein excretion rate. Because $\mathrm{YKL}-40$ levels had a high positive correlation with a variety of clinical factors, even after eliminating interference from other factors, we suggest that YKL-40 may play a role in a variety of metabolic disorders in patients with T2DM and chronic complications. Among the many factors, maximum ACR correlated with YKL-40. This study indicated that YKL-40 might be related to the development of DN. However, whether YKL-40 can be used as a new marker for diagnosing and monitoring DN requires further investigation.

\section{Conflicts of interest}

The authors declare no conflict of interest.

\section{ACKNOWLEDGMENTS}

Research supported by grants from the Liaoning Province Science and Technology Department of the Science and Technology Project (\#201131), the Shenyang Scientific and Technological Projects (\#201121), and the Liaoning Province "Hundred Talents Project" (\#2011377). 


\section{REFERENCES}

Batinic K, Höbaus C, Grujicic M, Steffan A, et al. (2012). YKL-40 is elevated in patients with peripheral arterial disease and diabetes or pre-diabetes. Atherosclerosis 222: 557-563.

Brix JM, Höllerl F, Koppensteiner R, Schernthaner G, et al. (2011). YKL-40 in type 2 diabetic patients with different levels of albuminuria. Eur. J. Clin. Invest. 41: 589-596.

Chakaroun R, Raschpichler M, Klöting N, Oberbach A, et al. (2012). Effects of weight loss and exercise on chemerin serum concentrations and adipose tissue expression in human obesity. Metabolism 61: 706-714.

Cohen-Bucay A and Viswanathan G (2012). Urinary markers of glomerular injury in diabetic nephropathy. Int. J. Nephrol. 2012: 146987.

El-Mesallamy HO, Mostafa AM, Amin Al and El Demerdash E (2011). The interplay of YKL-40 and leptin in type 2 diabetic obese patients. Diabetes Res. Clin. Pract. 93: e113-e116.

Horbinski C, Wang G and Wiley CA (2010). YKL-40 is directly produced by tumor cells and is inversely linked to EGFR in glioblastomas. Int. J. Clin. Exp. Pathol. 3: 226-237.

Kanasaki K, Taduri G and Koya D (2013). Diabetic nephropathy: the role of inflammation in fibroblast activation and kidney fibrosis. Front. Endocrinol. 4: 7.

Kastrup J, Johansen JS, Winkel P, Hansen JF, et al. (2009). High serum YKL-40 concentration is associated with cardiovascular and all-cause mortality in patients with stable coronary artery disease. Eur. Heart J. 30: 1066-1072.

Lee JH, Kim SS, Kim IJ, Song SH, et al. (2012). Clinical implication of plasma and urine YKL-40, as a proinflammatory biomarker, on early stage of nephropathy in type 2 diabetic patients. J. Diabetes Compl. 26: 308-312.

Ma WH, Wang XL, Du YM, Wang YB, et al. (2012). Association between human cartilage glycoprotein 39 (YKL-40) and arterial stiffness in essential hypertension. BMC Cardiovasc. Disord. 12: 35.

Moresco RN, Sangoi MB, De Carvalho JA, Tatsch E, et al. (2013). Diabetic nephropathy: traditional to proteomic markers. Clin. Chim. Acta. 421: 17-30.

Nielsen AR, Erikstrup C, Johansen JS, Fischer CP, et al. (2008). Plasma YKL-40: a BMI-independent marker of type 2 diabetes. Diabetes 57: 3078-3082.

Ogawa D and Makino H (2012). Pathogenesis of diabetic nephropathy: the role of inflammation. Nihon Rinsho 5: 389-392.

Persson F, Rathcke CN, Gall MA, Parving HH, et al. (2012). High YKL-40 levels predict mortality in patients with type 2 diabetes. Diabetes Res. Clin. Pract. 96: 84-89.

Rathcke CN, Persson F, Tarnow L, Rossing P, et al. (2009). YKL-40, a marker of inflammation and endothelial dysfunction, is elevated in patients with type 1 diabetes and increases with levels of albuminuria. Diabetes Care 32: 323-328.

Røndbjerg AK, Omerovic E and Vestergaard $\mathrm{H}$ (2011). YKL-40 levels are independently associated with albuminuria in type 2 diabetes. Cardiovasc. Diabetol. 10: 54.

Thomsen SB, Rathcke CN, Zerahn B and Vestergaard H (2010). Increased levels of the calcification marker matrix Gla Protein and the inflammatory markers YKL-40 and CRP in patients with type 2 diabetes and ischemic heart disease. Cardiovasc. Diabetol. 9: 86. 